FZ-IKP(TH)-2000-30

\title{
Diffractive beauty photoproduction as a short distance probe of QCD pomeron
}

\author{
V. R. Zoller \\ Institute for Theoretical and Experimental Physics, Moscow 117218, Russia \\ E-mail: zoller@heron.itep.ru
}

\begin{abstract}
High-energy open beauty photoproduction probes the vacuum exchange at distances $\sim 1 / m_{b}$ and detects significant corrections to the BFKL asymptotics coming from the subleading vacuum poles. We show that the interplay of leading and subleading vacuum exchanges gives rise to the cross section $\sigma^{b \bar{b}}(W)$ growing much faster than it is prescribed by the exchange of the leading pomeron trajectory with intercept $\alpha_{\mathbf{I P}}(0)-1=\Delta_{\mathbf{I P}}=0.4$. Our calculations within the color dipole BFKL model are in agreement with the recent determination of $\sigma^{b \bar{b}}(W)$ by the $\mathrm{H} 1$ collaboration. The comparative analysis of diffractive photoproduction of beauty, charm and light quarks exhibits the hierarchy of pre-asymptotic pomeron intercepts which follows the hierarchy of corresponding hardness scales. We comment on the phenomenon of decoupling of soft and subleading BFKL singularities at the scale of elastic $\Upsilon(1 S)$-photoproduction which results in precocious color dipole BFKL asymptotics of the process $\gamma p \rightarrow \Upsilon p$.
\end{abstract}

In this communication we address the issue of open beauty photo- and electroproduction

$$
\gamma^{*} p \rightarrow b \bar{b} X
$$

at large values of Regge parameter

$$
x^{-1}=\frac{W^{2}+Q^{2}}{4 m_{b}^{2}+Q^{2}} \gg 1,
$$

where $W$ is the c.m.s. collision energy, $Q^{2}$ is the photon virtuality and the mass of beauty quark $m_{b}$ sets the natural scale of the process (1).

In color dipole (CD) approach the excitation of heavy flavors at small- $x$ is described in terms of interaction of small size quark-antiquark $b \bar{b}$ color dipoles in the photon. This makes the reaction (1) a sensitive probe of short distance properties of vacuum exchange in QCD. The interaction of color dipole $\mathbf{r}$ with the target proton is described by the beam, target and flavor independent color dipole-proton cross section $\sigma(x, \mathbf{r})$. The contribution of excitation of open beauty to photo-absorption cross section is given by color dipole factorization formula 11, 2, 3 3

$$
\sigma^{b \bar{b}}\left(x, Q^{2}\right)=\int_{0}^{1} d z \int d^{2} \mathbf{r}\left[\left|\Psi_{L}^{b \bar{b}}(z, \mathbf{r})\right|^{2}+\left|\Psi_{T}^{b \bar{b}}(z, \mathbf{r})\right|^{2}\right] \sigma(x, \mathbf{r})
$$


where $\left|\Psi_{L, T}^{b \bar{b}}(z, \mathbf{r})\right|^{2}$ is a probability to find in the photon the $b \bar{b}$ color dipole with the beauty quark carrying fraction $z$ of the photon's light-cone momentum. The well known result of [2] for the transverse $(\mathrm{T})$ and longitudinal (L) photons is

$$
\begin{gathered}
\left|\Psi_{T}^{b \bar{b}}(z, r)\right|^{2}=\frac{\alpha_{e m}}{6 \pi^{2}}\left\{\left[z^{2}+(1-z)^{2}\right] \varepsilon^{2} K_{1}(\varepsilon r)^{2}+m_{b}^{2} K_{0}(\varepsilon r)^{2}\right\} \\
\left|\Psi_{L}^{b \bar{b}}(z, r)\right|^{2}=\frac{2 \alpha_{e m}}{3 \pi^{2}} Q^{2} z^{2}(1-z)^{2} K_{0}^{2}(\varepsilon r),
\end{gathered}
$$

where $K_{0,1}(y)$ are the modified Bessel functions, $\varepsilon^{2}=z(1-z) Q^{2}+m_{b}^{2}$ and $m_{b}=4.7 \mathrm{GeV}$ is the $b$-quark mass. Here we focus on the open beauty photoproduction cross section. At $Q^{2} \ll m_{b}^{2}$ the eq.(3) takes the form

$$
\sigma^{b \bar{b}}(x) \simeq \frac{\alpha_{e m}}{6 \pi} \int d r^{2} m_{b}^{2}\left[\frac{2}{3} K_{1}\left(m_{b} r\right)^{2}+K_{0}\left(m_{b} r\right)^{2}\right] \sigma(x, r)
$$

Because for small dipoles $\sigma(x, r) \propto r^{2}$, the dipole size integration in (6) is well convergent at small $r$ and the integrand (6) has a peak at $r \simeq 1 / m_{b}$. At very high $Q^{2} \gtrsim 4 m_{b}^{2}$ the peak develops a plateau for dipole sizes in the interval $\left(m_{b}^{2}+Q^{2} / 4\right)^{-1} \lesssim r^{2} \lesssim m_{b}^{-2}$. So, for moderate photon virtualities $Q^{2} \ll 4 m_{b}^{2}$ excitation of open beauty probes (scans) the dipole cross section at a special dipole size $r_{S}, r_{S} \sim 1 / m_{b}$.

The CD cross section $\sigma(x, r)$ at $x \ll 1$ satisfies the CD BFKL equation

$$
\frac{\partial \sigma(x, r)}{\partial \log (1 / x)}=\mathcal{K} \otimes \sigma(x, r),
$$

with the kernel $\mathcal{K}$ [四]. The basis of the CD BFKL-Regge expansion for $\sigma(x, r)$ [5, 6]

$$
\sigma(x, r)=\sum_{n} \sigma_{n}(r)\left(\frac{x_{0}}{x}\right)^{\Delta_{n}} .
$$

form the solutions of the eigen-value problem

$$
\mathcal{K} \otimes \sigma_{n}=\Delta_{n} \sigma_{n}(r)
$$

with Regge behavior, $\sigma_{n}(x, r)=\sigma_{n}(r)\left(x_{0} / x\right)^{\Delta_{n}}$.

The properties of the CD BFKL equation and the choice of physics motivated boundary condition at $x_{0}=0.03$ were discussed in detail elsewhere [7, 8, 9, 10, 11], here we only recapitulate features relevant to the considered problem.

The leading eigen-function $\sigma_{0}(r)$ for ground state i.e., for the rightmost hard BFKL pole, is node free. The subleading eigen-function for excited state $\sigma_{n}(r)$ has $n$ nodes. We find $\sigma_{n}(r)$ numerically [9, 10], for the semi-classical analysis see Lipatov [12]. The intercepts follow to a good approximation the law $\Delta_{n}=\Delta_{0} /(n+1)$. Within our specific infrared regularization we find $\Delta_{0}=0.402, \Delta_{1}=0.220$ and $\Delta_{2}=0.148$ [9]. The node of $\sigma_{1}(r)$ is located at $r=r_{1} \simeq 0.056 \mathrm{fm}$ (see Fig.1), for larger $n$ the rightmost node moves to a somewhat larger $r=r_{1} \sim 0.1 \mathrm{fm}$. As we discussed elsewhere [9], for still higher solutions, $n \geq 3$, all intercepts are very small anyway, $\Delta_{n} \ll \Delta_{0}$, For this reason, for the purposes of practical phenomenology we can truncate expansion (11) at $n=3$ lumping in the term $n=3$ contributions of still higher singularities with $n \geq 3$ (see Fig. 1 ). The term $n=3$ is endowed with the effective intercept $\Delta_{3}=0.06$ [13]. The truncated expansion reproduces 
the numerical solution $\sigma(x, r)$ of CD BFKL equation (7) in the wide range of dipole sizes $10^{-3} \lesssim r \lesssim 10 \mathrm{fm}$ with accuracy $\simeq 10 \%$ even at moderately small $x$.

Each CD BFKL eigen-cross section $\sigma_{n}$ via equation

$$
f_{n}^{b}\left(Q^{2}\right)=\frac{Q^{2}}{4 \pi^{2} \alpha_{e m}} \int_{0}^{1} d z \int d^{2} \mathbf{r}\left[\left|\Psi_{L}^{b \bar{b}}(z, \mathbf{r})\right|^{2}+\left|\Psi_{T}^{b \bar{b}}(z, \mathbf{r})\right|^{2}\right] \sigma_{n}(r) .
$$

defines the corresponding eigen-structure function $(\mathrm{SF}) f_{n}^{b}\left(Q^{2}\right)$ and we arrive at the $\mathrm{CD}$ BFKL-Regge expansion for the beauty SF of the proton $(n=0,1,2,3$, soft)

$$
F_{2}^{b}\left(x, Q^{2}\right)=\frac{Q^{2}}{4 \pi^{2} \alpha_{e m}} \sigma^{b \bar{b}}\left(x, Q^{2}\right)=\sum_{n} f_{n}^{b}\left(Q^{2}\right)\left(\frac{x_{0}}{x}\right)^{\Delta_{n}} .
$$

Analytical parameterization for eigen-SF $f_{n}^{b}\left(Q^{2}\right)$ is presented in Appendix. Notice that numerically $r_{1}>r_{S}$ and the rightmost nodes of subleading eigen-cross sections $\sigma_{n}(r)$ are located to the right of the peak of the integrand (6) (see Fig.1). Consequently, in the calculation of open beauty eigen-SFs $f_{n}^{b}\left(Q^{2}\right)$ one scans the eigen-cross section in between of the first and the second node. Hence, subleading $f_{n}^{b}\left(Q^{2}\right)$ which are negative valued in a wide range of $Q^{2}$. This point is illustrated in Fig. 2 in which the subleading BFKL-to-rightmost BFKL and soft-pomeron-to-rightmost BFKL ratio of eigen-SFs $r_{n}\left(Q^{2}\right)=f_{n}^{b}\left(Q^{2}\right) / f_{0}^{b}\left(Q^{2}\right)$ is shown. Because a probability to find large color dipoles in the photon decreases rapidly with the quark mass, the contribution from energy-independent soft-pomeron exchange to open beauty excitation is very small down to $Q^{2}=0$.

We comment first on the results on $\sigma^{b \bar{b}}(W)$. Because the CD BFKL-Regge expansion for color dipole-dipole cross section has already been fixed from the related and highly successful phenomenology of light flavor and charm contributions to the proton SF [6, 9, 10, 11] the CD BFKL predictions for the beauty SF of the proton are parameter free.

In Fig.3 our evaluation of the $W$-dependence of open charm (upper solid curve) and open beauty photoproduction cross sections (lower solid curve) is confronted to the HERA data [14, 15]. The H1 analysis of the data on $\sigma(\gamma p \rightarrow b \bar{b} X) \equiv \sigma^{b \bar{b}}$ for the collision energy in the range $94<W<266 \mathrm{GeV}$ with $\langle W\rangle \simeq 180 \mathrm{GeV}$ gives 15

$$
\sigma^{b \bar{b}}=111 \pm 10_{-11}^{+16} \pm 17 n b .
$$

The CD BFKL-Regge approach results in (Fig.3)

$$
\sigma^{b \bar{b}} \simeq 81 n b
$$

at $W \simeq 180 \mathrm{GeV}$. For an alternative interpretation of beauty photoproduction see [16]. We differ from [16] in estimate of the effect of $\log (1 / x)$-evolution. The latter seems to be strongly underestimated in [16].

As we have emphasized above, the characteristic feature of the QCD pomeron dynamics at distances $\sim m_{b}^{-1}$ is large negative valued contribution to $\sigma^{b \bar{b}}$ coming from subleading BFKL singularities. Consequences of this observation for the exponent of the energy dependence of the cross section

$$
\sigma^{b \bar{b}}(W) \propto W^{2 \Delta_{\text {eff }}}
$$

are quite interesting. In terms of the ratio $r_{n}\left(Q^{2}\right)$ (Fig.2) taken at $Q^{2} \ll 4 m_{b}^{2}$ and denoted by $r_{n}(0)$ the exponent $\Delta_{\text {eff }}$ reads $(\mathrm{n}=1,2,3$, soft $)$

$$
\Delta_{\text {eff }}=\Delta_{0} \frac{1+\Delta_{0}^{-1} \sum_{n} \Delta_{n} r_{n}(0)\left(x_{0} / x\right)^{\Delta_{n}-\Delta_{0}}}{1+\sum_{n} r_{n}(0)\left(x_{0} / x\right)^{\Delta_{n}-\Delta_{0}}}
$$


Because all coefficients $r_{n}(0)$ in eq.(13) are negative, except $r_{\text {soft }}(0)>0$, at HERA energies the effective intercept overshoots the asymptotic value $\Delta_{\mathbb{P}} \equiv \Delta_{0}=0.402$ (see Fig.4). At still higher collision energies both the soft and subleading hard BFKL exchanges become rapidly Regge suppressed. This results in decreasing $\Delta_{\text {eff }}$ down to $\Delta_{\mathbf{I P}}$.

For comparison, in photoproduction of open charm which scans the color dipole cross section at distances $\sim 1 / m_{c}$, in the vicinity of the rightmost node (see Fig.1), there is a strong cancellation between soft and subleading contributions to $\sigma^{c \bar{c}}(W)$ 11, 13. Consequently, for this dynamical reason in open charm photoproduction we have an effective one-pole picture and the effective pomeron intercept $\Delta_{\text {eff }} \simeq \Delta_{\mathbb{P}}$.

In photoproduction of light flavors the CD cross section $\sigma_{n}(r)$ is close to the saturation regime $\sigma_{n}(r) \propto$ const and all subleading and soft terms of the CD BFKL-Regge expansion are positive valued and numerically important (see [6] for more details). This is the dynamical reason for smallness of a pre-asymptotic pomeron intercept in photoproduction of light flavors (see Fig.4). Notice that it must not be taken at face value for $W \sim 1 \mathrm{TeV}$ because of likely strong absorption corrections. However, the hierarchy of pre-asymptotic intercepts which brings to light the internal dynamics of leading-subleading cancellations at different hardness scales should withstand unitarity effects.

In Fig. 5 we presented our predictions for the beauty structure functions in DIS on protons as a function of the Bjorken variable $x_{B j}=Q^{2} /\left(W^{2}+Q^{2}\right)$. The solid curve corresponds to the complete expansion (11) while the long-dashed curve is the leading hard pole approximation, $F_{2}^{b}\left(x, Q^{2}\right) \simeq f_{0}^{b}\left(Q^{2}\right)\left(x_{0} / x\right)^{\Delta_{0}}$. In agreement with the nodal structure of subleading eigenSFs the latter over-predicts $F_{2}^{b}$ significantly because the negative valued contribution from subleading hard BFKL exchanges overtakes the soft-pomeron exchange, see Fig. 2, and the background from subleading hard BFKL exchanges is substantial for all $Q^{2}$. We do not stretch the theoretical curves to $x>x_{0}=0.03$ beyond the applicability region of CD BFKL-Regge expansion (11).

Recently the cross section of elastic $\Upsilon(1 S)$ meson photoproduction has been measured at HERA [17]. Quarks in $\Upsilon$ meson are nonrelativistic and $|\gamma\rangle \propto m_{b} K_{0}\left(m_{b} r\right)$. The forward $\gamma \rightarrow$ $\Upsilon$ transition matrix element $\left\langle\Upsilon\left|\sigma_{n}(r)\right| \gamma\right\rangle$ is controlled by the product $\sigma_{0}(r) K_{0}\left(m_{b} r\right)$ [18] and the amplitude of elastic of $\Upsilon(1 S)$ photoproduction is dominated by the contribution from the dipole sizes $r \sim r_{\Upsilon}=A / m_{\Upsilon}$ with $A=5$. The crucial observation is that at distances $r \sim r_{\Upsilon}$ cancellation between soft and subleading contributions to the elastic photoproduction cross section results in the exponent $\Delta$ in

$$
\left.\frac{d \sigma(\gamma p \rightarrow \Upsilon p)}{d t}\right|_{t=0} \propto W^{4 \Delta}
$$

which is very close to $\Delta_{\mathbf{I P}}, \Delta=0.38$ [19]. This observation appears to be in agreement with the cross section rise observed by ZEUS\&H1 [17.

Appendix. For the practical applications it is convenient to have analytical parameterization for eigen-SFs $f_{n}^{b}\left(Q^{2}\right)$, which for the rightmost hard BFKL pole $n=0$ is of the form

$$
f_{0}^{b}\left(Q^{2}\right)=\frac{a R^{2} Q^{2}}{1+R^{2} Q^{2}}\left[1+c \log \left(1+r^{2} Q^{2}\right)\right]^{\gamma}, \gamma=\frac{4}{3 \Delta_{0}}
$$

where $a=3.877 \cdot 10^{-2}, R^{2}=1.036 \cdot 10^{-2} \mathrm{GeV}^{-2}, c=0.1482, r^{2}=1.004 \cdot 10^{-2} \mathrm{GeV}^{-2}$. For the subleading pole with $n=1$ the eigen-SF $f_{1}^{b}\left(Q^{2}\right)$ is of the form (15) with $a=-4.684 \cdot 10^{-3}$, $R^{2}=1.352 \cdot 10^{-2} \mathrm{GeV}^{-2}, c=0.2002, r^{2}=1.400 \cdot 10^{-2} \mathrm{GeV}^{-2}, \gamma=4 \delta / 3 \Delta_{0}$ and $\delta=1.621$. 
For still higher $n$ we have

$$
f_{2}^{b}\left(Q^{2}\right)=a f_{0}\left(Q^{2}\right)\left(1-z / z_{1}\right)\left(1+z / z_{2}\right) /\left(1+z / z_{3}\right)
$$

where $z=\log \left(1+r^{2} Q^{2}\right)^{\gamma}, \gamma=1.935, r^{2}=5.727 \cdot 10^{-2} \mathrm{GeV}^{-2}, a=-0.153, z_{1}=47.46$, $z_{2}=11.56, z_{3}=4944.7$ and

$$
f_{3}^{b}\left(Q^{2}\right)=a f_{0}\left(Q^{2}\right)\left(1-z / z_{1}\right)\left(1-z / z_{2}\right)
$$

where $z=\left[1+c \log \left(1+r^{2} Q^{2}\right)\right]^{\gamma}-1, \gamma=4 \delta / 3 \Delta_{0}, \delta=1.362, c=4.92 \cdot 10^{-2}, r^{2}=5.002 \cdot 10^{-3}$ $\mathrm{GeV}^{-2}, a=-0.3386, z_{1}=0.6844$ and $z_{2}=3.03$. The soft component of the beauty $\mathrm{SF}$ with $\Delta_{\text {soft }}=0$ was derived from eq.(3) with $\sigma_{\text {soft }}(r)$ taken from 19.

Acknowledgments: The author is grateful to J.Speth for hospitality at IKP(Theory) FZ-Juelich. This work was supported partly by the grant INTAS-97-30494.

\section{References}

[1] J.F.Gunion and D.E.Soper, Phys.Rev. D15 (1977) 2617

[2] N.N. Nikolaev and B.G. Zakharov, Z. Phys. C49 (1991) 607; J.Exp.Theor.Phys. 78 (1994) 598

[3] A.H.Mueller, Nucl.Phys. B415 (1994) 373

[4] N.N. Nikolaev, B.G. Zakharov, V.R. Zoller, JETP Letters 59 (1994) 8

[5] N.N. Nikolaev, B.G. Zakharov, V.R. Zoller, JETP 105 (1994) 1498

[6] N.N. Nikolaev, J. Speth and V.R. Zoller, Phys.Lett. B473 (2000) 157

[7] N.N.Nikolaev and B.G.Zakharov, Phys. Lett. B333 (1994) 250

[8] N.N. Nikolaev, B.G. Zakharov, Phys. Lett. B327 (1994) 157.

[9] N.N. Nikolaev, B.G. Zakharov and V.R. Zoller,JETP Lett. 66 (1997) 138

[10] N.N. Nikolaev and V.R. Zoller, JETP Lett. 69 (1999) 103

[11] N.N. Nikolaev and V.R. Zoller,JETP Lett. 69 (1999) 187

[12] L.N.Lipatov, Sov. Phys. JETP 63 (1986) 904.

[13] N.N. Nikolaev and V.R. Zoller, ITEP-PH-6-99, FZ-IKP-TH-1999-34, hep-ph/0001084

[14] ZEUS Coll., M.Derrick et al. Phys.Lett. B349 (1995) 225; H1 Coll., S.Aid, Nucl.Phys. B472 (1996) 32;

[15] Th. Kuhr, Open Beauty Cross Section at HERA, Talk given at DIS2000, April 2000

[16] S.Frixione, Nucl.Phys.Proc.Suppl. 79 (1999) 399; S.Frixione, M.L.Mangano, P.Nason and G.Ridolfi, Nucl.Phys. B431 (1994) 453 
[17] ZEUS Collab., J.Breitweg et al., Phys.Lett. B437 (1998) 432; H1 Collab., C.Adloff et al., Phys.Lett. B483 (2000) 23

[18] N.N.Nikolaev, Comments on Nucl. Part. Phys 21 (1992) 41; B.Z.Kopeliovich, J.Nemchik, N.N.Nikolaev and B.G.Zakharov, Phys.Lett. 309 (1993) 179; ibid. 324 (1994) 469; J.Nemchik, N.N.Nikolaev and B.G.Zakharov, Phys.Lett. 341 (1994) 228

[19] J. Nemchik, N.N. Nikolaev, E. Predazzi, B.G. Zakharov and V.R. Zoller, JETP 86 (1998) 1054 


\section{Figure captions}

Fig.1 The CD BFKL eigen functions plotted as $\sigma_{n}(r) / r$ for $\mathrm{n}=0,1,2$. The background term, $n=3$, which is a combination of higher CD BFKL solutions with $n \geq 3$ is also shown.

Fig.2 The subleading hard-to-rightmost hard and soft-pomeron-to-rightmost hard ratio of eigen-structure functions $r_{n}=f_{n}^{b}\left(Q^{2}\right) / f_{0}^{b}\left(Q^{2}\right)$ as a function $Q^{2}$.

Fig.3 Predictions from CD BFKL-Regge factorization for the heavy flavor $Q \bar{Q}$ photoproduction cross section. The lower curve is a result of the complete CD BFKL-Regge expansion for $\sigma^{b \bar{b}}(W)$. The upper curve corresponds to $\sigma^{c \bar{c}}(W)$ calculated with $m_{c}=1.3$ $\mathrm{GeV}$. The data points are from H1\&ZEUS HERA experiments 14, 15.

Fig.4 The W-dependence of the exponent $\Delta_{\text {eff }}$ in parameterization of the photoproduction cross section $\sigma^{q \bar{q}}(W) \propto W^{2 \Delta_{\text {eff }}}$ for different quark flavors.

Fig.5 Prediction from CD BFKL-Regge factorization for the beauty structure function of the proton $F_{2}^{b}\left(x, Q^{2}\right)$ as a function of the Bjorken variable $x_{B j}$. 


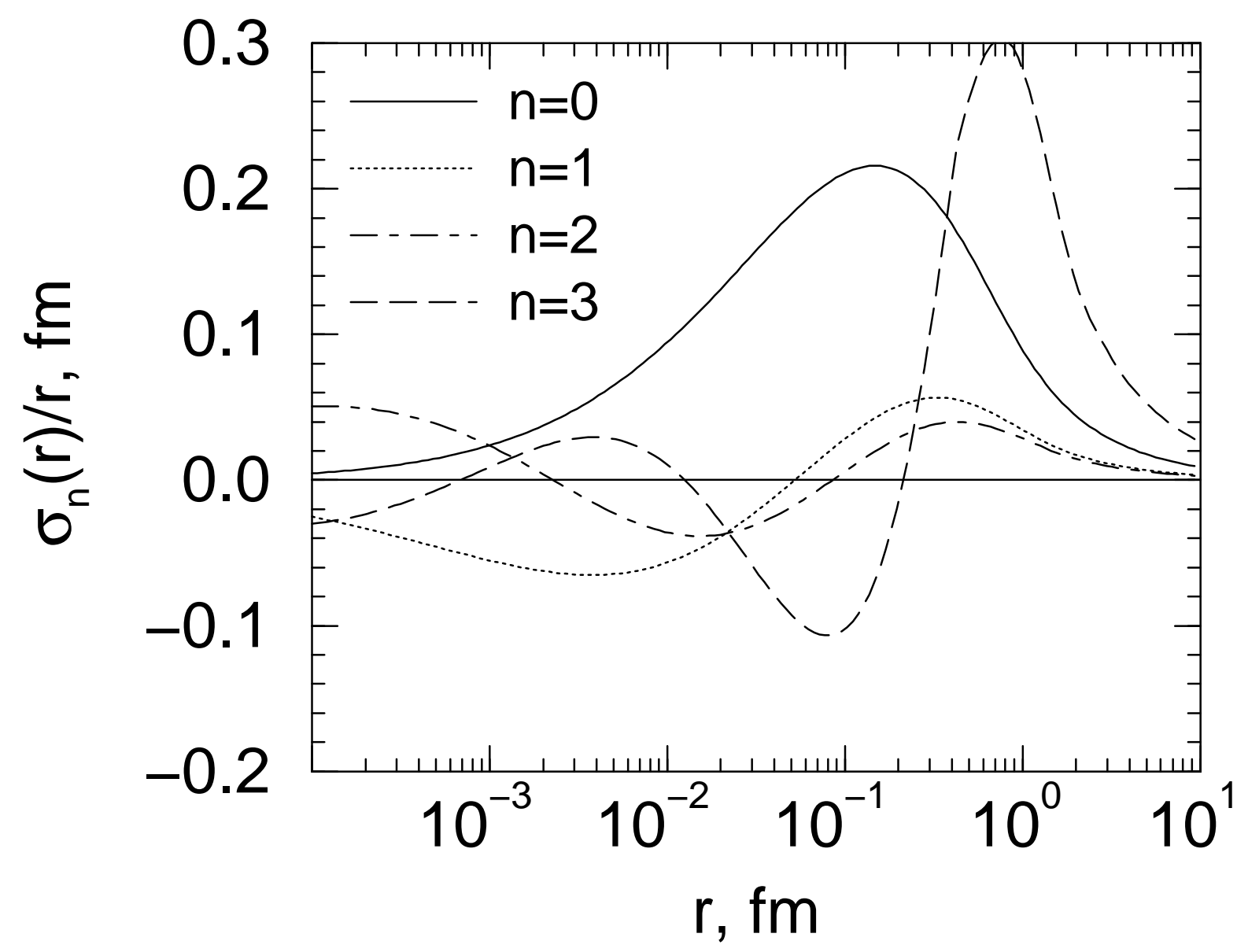




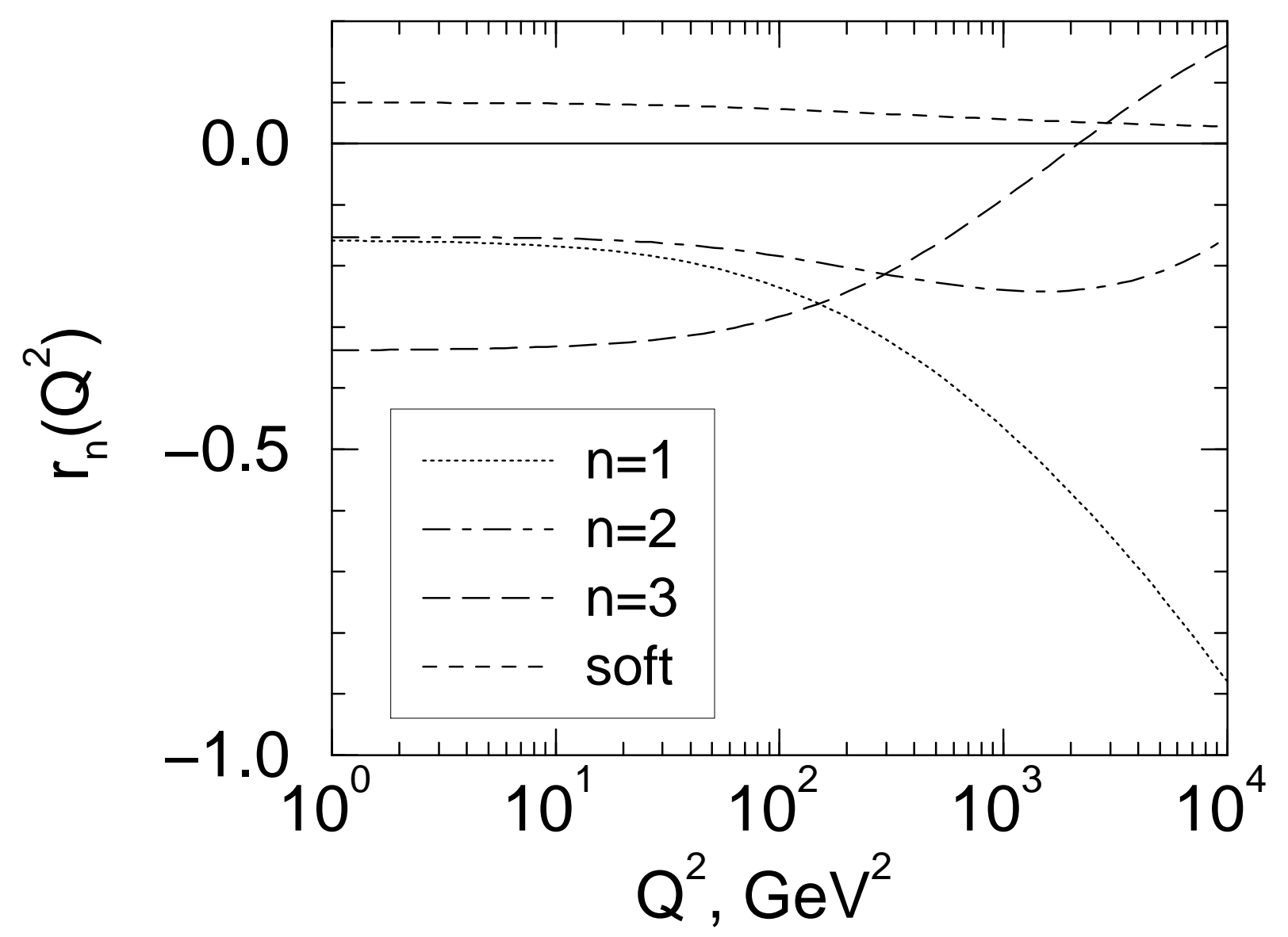




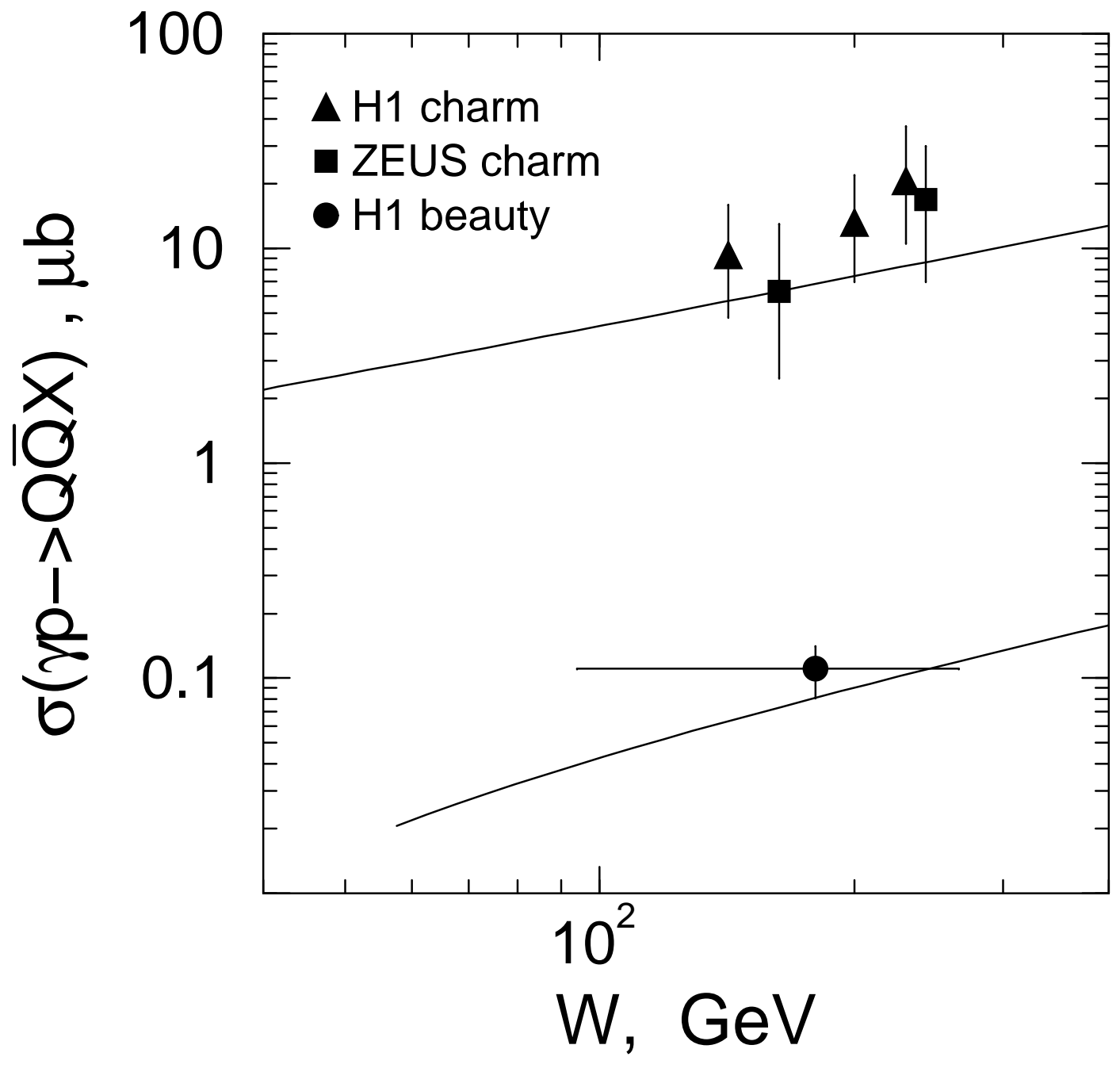




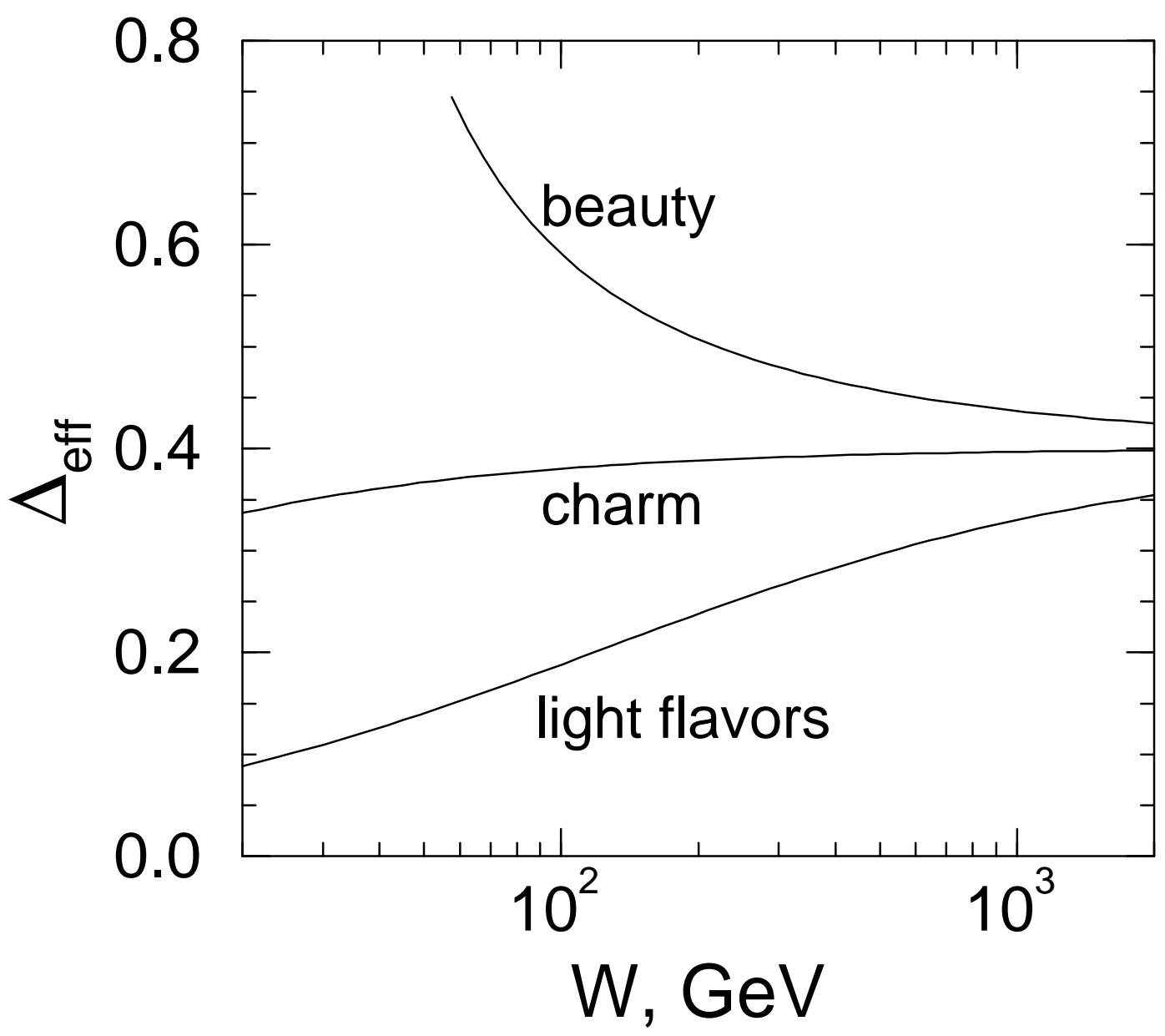




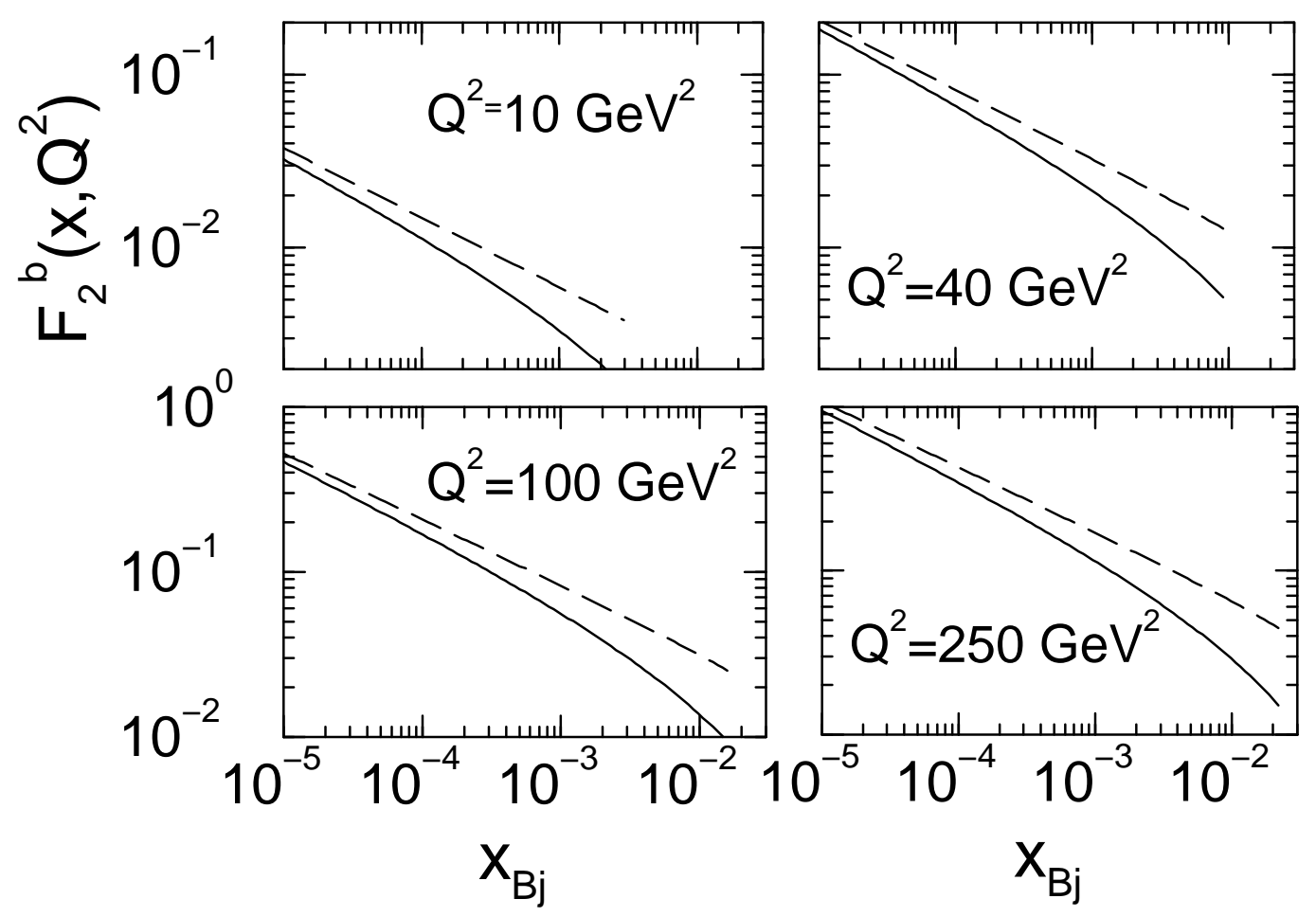

